\title{
Cpy(a/t)(q/w)e D-Hexapeptides Bind CUG Repeats and Rescue Phenotypes of Myotonic Dystrophy Myoblasts and A Drosophila Model of the Disease
}

Ariadna Bargiela ( $\square$ ariadna.bargiela@uv.es )

University of Valencia

Ruben Artero

University of Valencia

Roger Estrada-Tejedor

Ramon Llull University

Anna Rapisarda

University of Valencia

Enrique Garcia-España

University of Valencia

Beatriz Llamusi

Arthex Biotech S.L

Manuel Perez-Alonso

University of Valencia

Isabel Pont

University of Valencia

\section{Research Article}

Keywords:

Posted Date: December 28th, 2020

DOI: https://doi.org/10.21203/rs.3.rs-124856/v1

License: (1) (1) This work is licensed under a Creative Commons Attribution 4.0 International License.

Read Full License 


\section{Abstract}

In Myotonic Dystrophy type 1 (DM1), a non-coding CTG repeats rare expansion disease; toxic doublestranded RNA hairpins sequester the RNA-binding proteins Muscleblind-like 1 and 2 (MBNL1 and 2) and trigger other DM1-related pathogenesis pathway defects. In this paper, we characterize four D-amino acid hexapeptides identified together with abp1, a peptide previously shown to stabilize CUG RNA in its singlestranded conformation. With the generalized sequence $\operatorname{cpy}(\mathrm{a} / \mathrm{t})(\mathrm{q} / \mathrm{w}) \mathrm{e}$, these related peptides improved three MBNL-regulated exon inclusions in DM1-derived cells. Subsequent experiments showed that these compounds generally increased the relative expression of MBNL1 and its nuclear-cytoplasmic distribution, reduced hyperactivated autophagy, and increased the percentage of differentiated (Desminpositive) cells in vitro. All peptides rescued atrophy of indirect flight muscles in a Drosophila model of the disease, and partially rescued muscle function according to climbing and flight tests. Investigation of their mechanism of action supports that all four compounds can bind to CUG repeats with slightly different constant affinities, but binding did not strongly influence the secondary structure of the toxic RNA in contrast to abp1. Finally, molecular modeling suggests a detailed view of the interactions of peptide-CUG RNA complexes useful in the chemical optimization of compounds.

\section{Introduction}

Myotonic dystrophy type 1 (DM1; OMIM \#160900) is a rare autosomal dominant disease with symptoms that typically affect the musculoskeletal system, with degenerative muscle atrophy and myotonia (or muscle hyperexcitability), heart conduction defects, and cognitive involvement, which combined with several other multisystemic alterations severely affect the life expectancy and quality of life of patients ${ }^{1}$. DM1 originates from an abnormal expansion of an unstable CTG trinucleotide repeat in the 3'untranslated region of the $D M 1$ protein kinase $(D M P K)$ gene ${ }^{2}$. Expanded CTG repeats are transcribed but not translated and get retained in the cell nucleus where mutant transcripts accumulate, forming foci that sequester proteins of the Muscleblind-like (MBNL) family of alternative splicing regulators, among other molecular consequences ${ }^{3}$. The CUG expansions fold into metastable hairpin structures that facilitate the binding and sequestration of nuclear factors, among which MBNL1 is the most relevant in this work. Specifically, it has been shown that compound loss of $M b n / 1$ and $M b n / 2$ in mice reproduce several DM1 symptoms ${ }^{4}$. At the same time, Mbnl1 overexpression rescues DM1 phenotypes in a mouse model that expresses CTG repeats throughout the skeletal muscles ${ }^{56}$. Human MBNL1 proteins are tissue-specific $\mathrm{CCCH}$ zinc finger factors with crucial roles in the regulation of alternative splicing and alternative polyadenylation during development, in which it promotes a switch from fetal to adult patterns in a wide number of transcripts ${ }^{789}$. Although lack of MBNL1 function is one of the main molecular hallmarks of DM1 myopathy, many additional molecular contributors have been reported ${ }^{10}$. Hyperactivated GSKbeta and autophagy have been proposed to contribute to muscle atrophy in DM1 by stabilizing a repressive form of CELF1 alternative splicing regulator in the nucleus and downregulation of miR-7, a master regulator of autophagy, respectively ${ }^{11} 12,13$. Although most therapeutic strategies have focussed on degrading the expanded CUG RNA or preventing MBNL sequestration by the toxic RNA, with small 
molecules or oligonucleotide-based approaches ${ }^{14,15}$, direct upregulation of endogenous MBNL1 levels is becoming accepted as a complementary approach ${ }^{16} 1718$.

Drosophila is one of the experimentation animals used to model DM1 by expressing non-coding CTG repeat expansions to the insect muscles, brain, and heart to reproduce critical DM1 molecular defects and test candidate therapeutics 192021 . We previously targeted the expression of 480 interrupted CTG repeats to the Drosophila mushroom bodies, which are a pair of brain structures in insects. This generates a semilethal phenotype at the pupal stage used to screen a positional scanning synthetic combinatorial library of D-amino-acid hexapeptides that identified 16 candidate peptides, of which Abp1 (ppyawe) was characterized in more detail ${ }^{22}$. Our current study addressed the characterization of the remaining 15 peptides in a secondary screen in a DM1-derived cell model of the disease and identified four closely related peptides that improved cell and Drosophila DM1 phenotypes by directly binding to the CUG RNA.

\section{Results}

\section{Four related peptides rescue MBNL-dependent missplicing events in DM1 myoblasts.}

Immortalized human DM1 muscle cell lines display disease-associated molecular features such as nuclear RNA aggregates and alternative splicing defects ${ }^{23}$. DM1-related phenotypes can be used as readouts to screen candidate therapeutics in vitro for effects on RNA toxicity associated with the DM1 mutation. MBNL protein depletion explains most aberrant splicing patterns observed in DM1 242526 . Thus, we established three splicing events typically altered in DM1 as screening criteria for the $15 \mathrm{D}$ amino-acid hexapeptides previously identified (Supplementary Table 1) ${ }^{22}$ : the inclusion of exon 5 of cardiac troponin T gene (cTNT; Entrez ID: 7139) and the exclusion of exon 78 of the dystrophin gene ( $D M D$; Entrez ID: 1756), both MBNL1-dependent and the exclusion of exon 23 of spectrin alpha nonerythrocytic gene (SPTAN1; Entrez ID: 6709), which is MBNL2-dependent ${ }^{27}$. Immortalized control and DM1 fibroblasts were transdifferentiated into myoblasts for $48 \mathrm{~h}$. After that, they were incubated two more days with $10 \mu \mathrm{M}$ of each peptide dissolved in myoblast differentiation medium (MDM). Semiquantitative RT-PCR evaluated the activity of the peptides on the missplicing events. Despite most peptides being able to improve inclusion of at least one of the alternative exons, only peptides cpyaqe (79), cpyawe (80), cpytqw (81), and cpytwe (82) rescued all of them (Fig. 1a-d). Notably, the four peptides shared 4 out of 6 amino acids, and only the fourth and the fifth positions changed, generating the consensus sequence $\operatorname{cpy}(\mathrm{a} / \mathrm{t})(\mathrm{q} / \mathrm{w})$ e, which strongly suggests a structure-function relationship. Treatment with these peptides did not change the inclusion of exon 8 of the $C A P Z B$ gene, regulated by CELF1 ${ }^{28}$, nor exon 19 of the DLG1 gene, which remains unchanged in DM1 patients ${ }^{29}$, suggesting a specific effect on the regulatory factors MBNL1 and 2 in the disease (Fig. 1e-g). Finally, we tested whether such activity was (CUG) exp -specific or not; thus, we treated control cells with $10 \mu \mathrm{M}$ of peptide 80 and quantified their activity on the alternative exons of $C T N T$ and SPTAN. The peptide produced no significant change, suggesting that its activity depended on the presence of the mutation that causes DM1 (Fig. 1h-j). Peptides 79, 80, 81, and 82 were selected for further evaluation, including toxicity assays in control 
myoblasts (Fig. 1k) and fibroblasts (Supplementary Fig. 1). Peptides were not toxic even at $100 \mu \mathrm{M}$, which is ten times higher than the concentration at which they rescued missplicing.

\section{Candidate peptides enhance MBNL expression and its normal distribution in the cell.}

To understand what could trigger the rescue of the analyzed splicing events, we used cDNA from treated cells to perform quantitative PCR (qPCR) and detect any modification in the mRNA levels of MBNL 1 and MBNL2. We found that peptides $79,80,81$, and 82 doubled MBNL 1 expression while peptides 79 and 81 slightly increased MBNL2 mRNA amounts (Fig. 2ab). This finding was encouraging as the increase in MBNL1 and 2 gene expression has been proposed as a valid strategy to improve the clinical outcome of

DM1 5630161819 . However, at the protein level, only cells treated with the peptides 80 and 81 showed a statistically significant increase in MBNL1 levels by western blot compared to untreated DM1 cells (Fig. 2c, Supplementary Fig. 2). Finally, since the subcellular localization of MBNL1 is altered in DM1 19,30, we evaluated this phenotype using an anti-MBNL1 antibody. Immunofluorescence images confirmed that the MBNL1 signal was increased in the cytoplasm of the treated cells compared to the untreated and approached normal intensity and subcellular distribution (Fig. 2d-i). Taken together, these results indicate that the candidate peptides were able to target the upregulation of MBNL1. Therefore, we studied additional molecular phenotypes related to MBNL1 in the pathogenesis pathway.

\section{A 48-h treatment is sufficient to reduce the number of foci with no effect on the DMPK transcripts.}

A prime mechanism to enhance functional MBNL1 levels in cells is to prevent its sequestering into ribonuclear foci by either blocking its binding to CUG repeats or changing the secondary structure of the toxic RNA so it is less prone to bind to the protein ${ }^{15,22,31}$. We quantified the number of foci per nucleus to shed light on this problem, using fluorescence in situ hybridization with an RNA probe (Cy3-(CAG)7-Cy3) and an IN Cell Analyzer High-Content Cellular Analysis System to acquire images (Fig. 3a-h). The treatment with each of the peptides produced a significant change in the number of cells without foci, which increased, and in the percentage of foci per cell, which was significantly reduced (Fig. 3i,j). These changes occurred without altering the relative expression levels of DMPK transcripts (Fig. $3 \mathrm{k}$ ), half of which carry the expanded CUG triplets, suggesting the possibility of a direct interaction of the peptides with the RNA, with or without a subsequent influence on its secondary structure.

\section{Treated DM1 myoblasts reduce the differentiation delay.}

Symptoms of myotonia, muscle weakness, and muscular atrophy are the main features of DM1 ${ }^{1}$, and the molecular contributions to these symptoms are numerous ${ }^{10}$. One of them is a delay in the process of muscle differentiation, which can be quantified as a reduction in the fusion index after the induction of the fibroblasts to myoblasts transdifferentiation ${ }^{32} 33$. After incubating with MDM both the control and DM1 fibroblasts for four and seven days and treatment with the peptides for $48 \mathrm{~h}$, we carried out immunofluorescence with an anti-Desmin antibody and quantified Desmin-positive (differentiated) cells. While the percentage of terminally differentiated cells remained unchanged after four days in MDM 
medium, after seven days the percentage of Desmin-positive DM1 cells increased significantly upon treatment with peptides 79 and 81 and remained unchanged in the presence of a scrambled control peptide (Fig. 4a-i). The fusion index, however, did not significantly increase (Supplementary Fig. 3).

Another molecular mechanism contributing to muscle atrophy in DM1 is the activation of autophagy $12,13,34$. To check the autophagy status in DM1 cells after peptide treatments, we used the lysotracker reagent, which stains acidic lysosomes ${ }^{35}$. First, we confirmed that the level of autophagy of the diseased cells was considerably higher than that of healthy cells (Fig. 4j,k). Furthermore, we verified that the treatment with the peptide 80 caused a robust reduction in the signal associated with the lysosomal vesicles, indicating a recovery of the normal autophagy levels. Along the same lines, peptides 81 and 82 increased the number of cells devoid of autophagic vesicles around the nucleus (Fig. 4j-0). Thus, these observations indicate a general reduction in the autophagy pathway, which has previously been shown to contribute to muscle atrophy in Drosophila and human cells in vitro ${ }^{12,13,34}$.

\section{Candidate peptides rescue muscle atrophy of a Drosophila model of the disease.}

Rescue of two molecular phenotypes related to muscle atrophy in the cell model, namely delayed differentiation and hyperactivated autophagy, prompted us to verify if these peptides were also active in vivo in a muscle phenotype in Drosophila. In this model, the expression of toxic CUG repeats is controlled by the myosin heavy chain promoter and reproduces muscle phenotypes observed in humans ${ }^{13,36,37}$. After feeding the DM1 flies with $10 \mu \mathrm{M}$ of each peptide mixed with the food, we embedded the thorax of the flies to obtain cross-sections of indirect flight muscles. The quantification of the muscle area from these images showed a marked improvement in the atrophic phenotype in peptide-treated flies, bringing the muscle area to values very close to those observed in control flies (Fig. 5a-i)). Concomitant to muscle atrophy, model flies have reduced locomotor abilities, which in flies can be assessed through climbing, taking advantage of Drosophila's negative geotropism, and flight assays. First, we used 30 male flies for the climbing experiment to measure the height climbed by the flies in a given time. The results revealed a significant increase in the speed of the flies treated with the four peptides compared with DM1 flies fed with DMSO supplemented food or scrambled peptide controls (Fig. 5j). In flight tests, while there were no significant increases in the height of the landing distance (indicative of better flight capabilities), we found an increase in the percentage of flies capable of flying, especially in the case of peptide 82 treatment, where it reached statistical significance and almost doubled the value observed in controltreated DM1 flies ( $p=0.0044$, Fisher's exact test; Fig. $5 k$ ). In conclusion, the increase in the number of flies showing the ability to fly indicates a partial rescue of the Drosophila muscle function consistent with the increase in the IFM muscle area.

\section{The secondary structure of (CUG) ${ }_{23}$ RNA remains unchanged after candidate peptides binding.}

We used a Differential Scanning Fluorimetry (DSF) assay to monitor CUG RNA thermodynamics in the presence of increasing concentrations of candidate peptides. DSF is technique used to study the effect of compounds on RNA stability as RNA undertakes structural conversions upon thermal unfolding ${ }^{38}$. When 
RNA changes its structure the single stranded form increases the available binding sites for RiboGreen dye. We represented the first derivatives of normalized fluorescence of RiboGreen with an RNA probe containing 23 repeats of CUG versus temperature in the presence of concentrations of each hexapeptide ranging from 1 to $100 \mu \mathrm{M}$ (Fig. 6a,d,g,j). The titration with increasing concentrations of peptides 79,80 , and 81 only changed the height of the peak indicating interference in the intrinsic folding properties of the RNA probe rather than stabilization or destabilization of the RNA hairpins. Peptide 82, however, did slightly shift the curve peak towards lower temperatures, which meant that the interaction between the hexapeptide and the probe does not stabilize the single strain RNA conformation, which is in striking contrast with the proposed destabilization of CUG RNA by abp $1^{22}$. Taken together, the DSF experiments strongly support that the candidate peptides, at least 79,80 , and 81 , do not significantly modify the secondary structure of the CUG RNA.

\section{Candidate peptides interact with CUG RNA with similar affinities.}

A fluorescent indicator displacement (FID) assay was used to investigate the nature of the interaction between the candidate peptides and the toxic RNA. Thiazole orange (TO) is an asymmetric cyanine intercalator with little fluorescence when free in an aqueous solution but strong emission when forming complexes of different nature with nucleic acids. These characteristics can be exploited to study changes in the interaction between the dye and the nucleic acid of interest in response to external factors 39,40 . Specifically, the fluorescent reporter interacted with the (CUG) ${ }_{23}$ RNA probe resulting in fluorescent emission. By adding the peptide, it was possible to displace the TO, causing its fluorescence to decrease. In this way, by analyzing the fluorescence emission at different RNA-peptide ratios, the value of the peptide's affinity constant for the CUG sequence could be indirectly calculated. For all peptides, a progressive reduction in fluorescence was observed in response to increasing concentrations of each peptide (Fig. $6 \mathrm{~b}, \mathrm{e}, \mathrm{h}, \mathrm{k})$; in the case of peptide $79\left(\mathrm{k}_{\mathrm{log}}=5.273 \pm 0.007\right)$, the greatest decrease in fluorescence was observed, followed by peptide $80\left(k_{\log }=5.04 \pm 0.01\right)$ and the smallest change was observed with peptides 82 and 81 (respectively $k_{\text {log }}=4.74 \pm 0.02$ and $k_{\log }=4.65 \pm 0.02$ ) (Fig. 6c,f,i,l). However, it should be noted that in none of the cases did the level of fluorescence observed reach the characteristic values of a single-stranded RNA. This indicates that, although there was a clear interaction between the peptides and the RNA molecule, peptides did not interfere with the stability of the secondary structure of CUG RNA, which was consistent with the data generated by the DSF assay.

In parallel with the above experiments, we used the peptide showing the highest affinity for CUG repeats to investigate a potential direct interaction with MBNL1 proteins as an alternative mechanism of action since it might similarly prevent sequestration by the repeats. Double immunostaining with biotin-labeled peptide 79 revealed it accumulated in the cytoplasm of DM1 myoblasts, mainly in the perinuclear area, but no significant overlap was found with the MBNL1 protein signal (Supplementary Fig. 4). Thus, candidate peptides do not seem to interact physically with MBNL proteins, at least peptide 79.

\section{Study of the interaction mechanism by molecular docking.}


In molecular modeling, docking is a method that predicts the preferred orientation of one molecule to a second when they bind together to form a stable complex. Multiple docking studies were performed using Autodock VINA and Molecular Operating Environment (MOE) software to assess the preferred binding mechanism between hexapeptides and CUG repeats. Next, the results obtained with blind docking and guided docking techniques were compared; in the latter case, tests were carried out keeping the RNA rigid or admitting certain flexibility. The final docking protocol was validated by correlating the binding affinities predicted by docking (score) and FID results. According to the analysis, peptides 79 and 80 showed the most remarkable tendency to interact in the exposed part of the RNA (Fig. 7a,b). More in detail, peptide 79 was the only D-hexapeptide capable of recognizing two uracils of the two RNA chains by means of the two terminal amino acid residues. This observation could explain that it had the highest RNA binding constant affinity. Hexapeptide 80 would interact with the two strands of RNA but showing only the recognition of one uracil. According to the results, Trp would not be favorably available for interaction with the RNA backbone. This result is in agreement with the experimental data in which the presence of Trp did not contribute to the increase in the RNA binding constant (cpyaqe > cpyawe, cpytqe $\sim$ cpytwe). Although this conclusion could be due to a limitation of the simulation protocol used, which only contemplates slight flexibility of the RNA, it can be inferred that Trp would not interact by $\Pi \Pi^{-}$ stacking (attractive and non-covalent interactions between aromatic rings). As for the two peptides with the lowest experimental interaction energy, it was surprising that the binding mechanism obtained, both for peptides 81 and 82, only showed interaction with one strand of RNA (Fig. 7c,d). Of the two, 82 could interact with three consecutive nucleotides, providing a slight additional stabilization.

\section{Discussion}

In this paper, we report identifying four related peptides, with the sequence $c p y(a / t)(q / w) e$, that improved splicing and differentiation phenotypes in DM1-derived cells and reduced ribonuclear foci number through the direct binding to the repeats. Muscle area and functional defects in a Drosophila model also improved upon oral administration to the flies. We show that the candidate peptides bind CUG repeats with similar affinity but do not impinge on the secondary structure of the toxic RNA and propose a detailed mechanism of potential interactions between the peptides and the repeats. Although the peptides behaved similarly in many experimental conditions, they also showed differences. The explanation for these differences could lie in the sequence variations, which are responsible for molecular structural changes. Minimal structural changes can significantly interfere in the molecular interaction between peptides and their targets ${ }^{41}$. Despite some unspecific effects by the scrambled peptide in climbing assays, the relevance of the sequence is clearly demonstrated in Drosophila muscle area determinations and in cell experiments in which SC peptide did not affect cross-sectional muscle area (Fig. 5a), the number of foci per cell, percentage of cells without foci (Fig. 3) or cell differentiation (Fig. 4).

Peptide specificities are best illustrated as a spider graph, in which nine DM1-related parameters are simultaneously represented for each of the peptides using a semiquantitative scale that ranks peptides in each of these parameters (Fig. 8a). Thus, for example, while in terms of foci reduction peptide 81 shows 
the most activity, followed by 80,82 , and 79 , for cell differentiation the rank was peptide $81>79>82>80$. Indeed, since the higher the score, the better the rescue, we can integrate the area of the polynomial defined by each peptide to rank the overall therapeutic potential of peptides as $81>82>80>79$ (Fig. 8b). These images also help illustrate that peptide 81 exhibited the best corrective capacity in DM1 patient cells, while peptide 82 was more effective in the Drosophila disease model.

Thanks to their intrinsic property of high specificity for a target biomolecule and the use of strategies to improve stability and uptake, and to reduce toxicity, several non-natural peptides have already reached medical use ${ }^{42}$. Indeed, once identified, peptides with promising anti-DM1 potential can be further improved using various strategies to develop a lead compound suitable for clinical studies 424344 . Among the four candidate peptides, 81 is regarded as the most promising since it had the highest human cell activity and significant positive effects in Drosophila. The identified peptides are also very short, which in general terms can be expected to enhance distribution among biological tissues. In the hit-tolead process, some well-established strategies could improve the pharmacological characteristics of the peptides. For example, various studies have shown that the bioactive peptide conjugation with a cellpenetrating peptide considerably enhanced its activity ${ }^{45}$. This is particularly important for a peptide that must reach the muscles. Therefore, it would be of interest to conjugate our candidate peptides with the TAT fragment $\left({ }^{48} \mathrm{GRKKRRQRRR}{ }^{57}\right)$ or with a poly-Arginine peptide $\left(\operatorname{Arg}_{5-8}\right)$, either directly or through a spacer linker ${ }^{46}$. Other successful strategies are the conversion of the peptide with the desired biological activity into a peptoid, in which the side chain is connected to the nitrogen of the peptide backbone, instead of the a-carbon as in peptides, and other types of peptidomimetics, to improve stability and cellular uptake ${ }^{47}$.

Our molecular modeling study offers a detailed view of how peptides 79-81 might be interacting with CUG repeats. One observation is that, given the known flexibility of the RNA under study, it is not surprising that the observed interactions may show fluctuations. Even so, the results indicate that the two interactions established by peptide 81 with $\mathrm{C}$ and $\mathrm{G}$ are more labile than the three presented by peptide 82 , a fact that could explain its slightly higher binding constant. It is also observed that peptide 80 maintains its interaction with RNA in a significant way, although Trp is not capable of establishing a permanent interaction. Similarly, the interactions initially defined by peptide 79 with consecutive CUG uracils are labile but are recovered during the simulation. It is finally interesting to remark that peptides 79-81 start with a cysteine (c) and end with a glutamic acid (e), which is a structural feature conserved within the zinc fingers 2 and 4 of MBNL1, 2 and 3, and zinc finger 1 of MBNL2 and also show other coincidences with conserved amino acids within zinc fingers of these proteins in positions 4 (a) and 5 (q) (Supplementary Fig. 5). Interestingly, molecular modeling predicts the terminal residues of peptide 79 to interact with two $U$ in the double-stranded CUG RNA (Fig. 7a).

In brief, we propose that 79-81 peptides bind CUG repeats and prevent MBNL1 depletion with little or no change in the secondary structure of the CUG RNA. An increase in MBNL1 expression, perhaps as a consequence of the improved MBNL1 function itself, rescues MBNL1-dependent activities in both the cell 
nucleus and the cytoplasm. Enhanced MBNL1 levels and close-to-normal distribution in the cell brings about cell model rescues at the molecular and cytological levels. Despite the close similarity of 79-81 peptides with the previously identified abp $1^{22}, \operatorname{cpy}(\mathrm{a} / \mathrm{t})(\mathrm{q} / \mathrm{w}) \mathrm{e}$ and ppyawe, respectively, the mechanism abp1 used to prevent sequestration was the stabilization of CUG RNA in its single-stranded conformation compared to an apparent sterical hindrance by $79-81$. Together with abp 1 , these peptides offer relevant substrates for more focused medicinal chemistry studies towards developing therapies for myotonic dystrophy.

\section{Methods}

\section{Hexapeptides used}

D-amino acid hexapeptides used in this work (Supplementary Table 1) were purchased from GenScript (purity $>98 \%$ ) and were dissolved in $100 \%$ DMSO as stock solutions and stored at room temperature until use.

\section{Drosophila methods}

For the evaluation of compounds, we used a previously described recombinant Drosophila line in which a

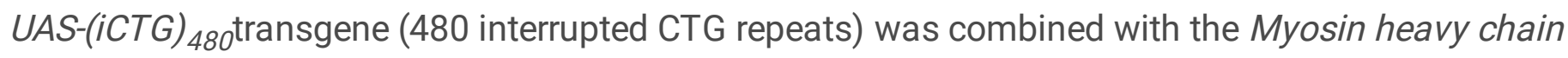
(Mhc)-Gal4 driver for continuous expression of the toxic RNA in the fly muscles, hereafter referred to as Rec- $2^{36}$. For the crosses, $20 \mathrm{w}$ females and 10 Rec 2 males were crossed and 12 females of the offspring were hand-collected and transferred to a tube containing $3 \mathrm{ml}$ of regular Drosophila medium supplemented with $10 \mu \mathrm{M}$ of each compound, or $0.01 \%$ of DMSO as a control. Flies were moved to a tube with fresh food (supplemented with compounds as described above) every other day for seven days when they were processed for muscle area determinations. All fly lines were maintained at $25^{\circ} \mathrm{C}$ on a standard day-night cycle. Climbing and flight assays were as described in ${ }^{19}$.

\section{Cross-sectional muscle area determination}

Analysis of the IFM area in Drosophila thoraces was performed as previously described ${ }^{48}$. Briefly, six thoraces of seven-day-old females were embedded in Epon. Semi-thin $1.5 \mu \mathrm{m}$-sections were obtained using an ultramicrotome (Ultracut E, Reichert-Jung and Leica). Images were taken at 100x magnification with a Leica DM2500 microscope (Leica Microsystems, Wetzlar, Germany). Five images containing IFMs per fly were converted into binary images using the ImageJ software, and the percentage of black pixels (corresponding to muscle) to the total number of pixels within a fixed-size frame was calculated.

\section{Cell culture and proliferation assay}

Unaffected (control) and patient-derived (DM1) cells were kindly provided by Dr. Furling (Institute of Myology, Paris) ${ }^{23}$. Fibroblast cells were grown in Dulbecco's Modified Eagle's Medium (DMEM) with 4.5 $\mathrm{g} / \mathrm{L}$ glucose, $1 \%$ penicillin and streptomycin (P/S), and 10\% fetal bovine serum (FBS; Sigma). To 
transdifferentiate fibroblasts into myoblasts, the cells were plated in muscle differentiation medium (MDM) containing DMEM with $4.5 \mathrm{~g} / \mathrm{L}$ glucose, $1 \% \mathrm{P} / \mathrm{S}, 2 \%$ horse serum, $1 \%$ apo-transferrin $(10 \mathrm{mg} / \mathrm{ml})$, $0.1 \%$ insulin $(10 \mathrm{mg} / \mathrm{ml})$, and $0.02 \%$ doxycycline $(10 \mathrm{mg} / \mathrm{ml})$. In all cases, the cells were grown at $37^{\circ} \mathrm{C}$ in a humidified atmosphere containing $5 \% \mathrm{CO}_{2}$.

For toxicity assays, cells were seeded at $10^{4} \mathrm{cell} / \mathrm{ml}$ in 96 -well plates differentiated for $96 \mathrm{~h}$ and treated with different concentrations of peptides $(10 \mu \mathrm{M}, 50 \mu \mathrm{M}, 75 \mu \mathrm{M}$, and $100 \mu \mathrm{M}) ; 48 \mathrm{~h}$ post-incubation, cell proliferation was measured using the CellTiter $96 \AA$ AQueous Non-Radioactive Cell Proliferation Assay (Promega) following the manufacturer's instructions. The $\mathrm{IC}_{10}$ and dose-response inhibition curves were calculated using non-linear least-squares regression, and absorbance levels were determined using a Tecan Infinite M200 PRO plate reader (Life Sciences).

The fusion index was defined as the percentage of nuclei within myotubes ( $>2$ myonuclei) regarding the total number of nuclei in each condition. The average number of total nuclei per myotube was determined by counting over 250 nuclei from randomly chosen Desmin-positive cells (5-7 micrographs).

\section{Immunofluorescence methods}

For immunofluorescence detections, myoblasts were fixed with 4\% PFA for $15 \mathrm{~min}$ at room temperature (RT) followed by several washes in $1 \times$ PBS. Cells were then permeabilized with PBS-T ( $0.3 \%$ Triton- $X$ in PBS) and blocked (PBS-T, 0.5\% BSA, 1\% donkey serum) for 30 min at RT, and incubated with primary antibody mouse anti-MBNL1 (1:200, ab77017, Abcam), anti-Desmin (1:200, ab8470, Abcam) or anti-LC3 (1:2000, ab243506, Abcam) at $4^{\circ} \mathrm{C}$ overnight. After several PBS-T washes, the cells were incubated for 1 $\mathrm{h}$ with a biotin-conjugated anti-mouse-IgG secondary antibody (1:200, Sigma-Aldrich) to detect antiMBNL1, anti-Desmin, or anti-LC3. The fluorescence signal was amplified with an Elite ABC kit (VECTASTAIN) for $30 \mathrm{~min}$ at RT, followed by PBS-T washes and incubation with streptavidin-FITC (1:200, Vector) to detect anti-MBNL1 or anti-Desmin, for 45 min at RT. After several washes with PBS, the cells were mounted with a VECTASHIELD $®$ mounting medium containing DAPI (Vector) to detect the nuclei.

For MBNL1 and 79-biotin peptide co-localization assay, myoblasts were fixed with 4\% PFA for 15 min at RT followed by several washes in $1 \times$ PBS. Cells were then permeabilized with PBS-T and blocked (PBS-T, $0.5 \%$ BSA, $1 \%$ donkey serum) for 30 min at RT and incubated with primary antibody mouse anti-MBNL1 $(1: 200, a b 77017, A b c a m)$ at $4^{\circ} \mathrm{C}$ overnight. After several PBS-T washes, the cells were incubated for $1 \mathrm{~h}$ with a FITC-conjugated anti-mouse-lgG secondary antibody (1:200, Sigma-Aldrich) to detect anti-MBNL1. The fluorescence signal of the 79-biotin peptide was amplified with an Elite ABC kit (VECTASTAIN) for 30 min at RT, followed by PBS-T washes and incubation with streptavidin-TEXAS RED (1:200, Vector) for 45 min at RT. After several washes with PBS, the cells were mounted with a VECTASHIELD ${ }^{8}$ mounting medium containing DAPI (Vector).

For the detection of lysosomes, CNT and DM1 myoblasts were treated as described above for immunofluorescence but incubated with $100 \mathrm{nM}$ LysoTracker RED-DND99 and $5 \mu \mathrm{g} / \mathrm{ml}$ Hoechst 33258 
(Invitrogen and Sigma-Aldrich, respectively) at $37^{\circ} \mathrm{C}$ for $30 \mathrm{~min}$, and were mounted using fluorescence mounting medium (Dako, Glostrup, Denmark). Images were taken at 400x magnification using a fluorescence microscope Leica DM4000 B LED.

For fluorescent in situ hybridization, fibroblasts were seeded into 96 well Cell Culture Plate $\left(1 \times 10^{5}\right.$ cells per well) and treated with peptides. In situ detection was performed as previously described ${ }^{49}$. Images were taken and analyzed using an IN Cell Analyzer 2200 Imaging System (GE Healthcare).

\section{RNA extraction, RT-PCR, and quantitative real-time PCR (qRT-PCR)}

Total RNA from human myoblasts was isolated using TRIreagent (Sigma). One microgram of RNA was digested with DNase I (Invitrogen) and reverse-transcribed with SuperScript II (Invitrogen) using random hexanucleotides; cDNA was used in a standard PCR reaction with GoTaq polymerase (Promega). Specific primers were used to analyze the alternative splicing of DMD, CTNT, SPTAN1, CAPZB, and DLG1 in control and DM1 human myoblasts ${ }^{12}$. GAPDH was used as endogenous controls. PCR products were quantified using ImageJ software $(\mathrm{NIH})$. For multiplex qRT-PCR we used $1 \mathrm{ng}$ of human myoblast cDNA as template using the QuantiFast Probe PCR Kit reagent. Commercial TaqMan probes (Qiagen) were used to detect human MBNL 1,MBNL2, and DMPK expression levels with an Applied Biosystems StepOnePlus Real-Time PCR System. Results from myoblasts were normalized to GAPDH (VIC-labelled probe; Integrated DNA Technologies). Expression relative to the endogenous gene and control group was calculated using the 2 $\Delta \Delta \mathrm{Ct}$ method. Exon inclusion data come from at least three biological replicates, while real-time PCR was done with three technical replicates from each of three independent biological samples.

\section{Western blotting}

Procedure was performed as previously described ${ }^{30}$.

\section{Differential scanning fluorimetry (DSF) and fluorescent indicator displacement (FID) assays}

DSF experiments were performed to understand the peptides' interaction with the double-stranded CUG $\mathrm{RNA}^{38}$. The experiment was performed using a StepOnePlus Real-Time PCR system (Life Technologies) with the melting curve software to measure the fluorescence intensity. A MicroAmp ${ }^{\circledR}$ Fast Optical 96-well plate (Life Technologies) was used with $50 \mu$ l of solution per well. The RiboGreen dye was used at a final concentration of $300 \mathrm{nM}$, whereas the synthetic double-stranded CUG RNA (12× CUG) was used at a final concentration of $600 \mathrm{nM}$. For each compound (in this case the four peptides and DMSO), four technical replicates were performed and, for each peptide, four different concentrations ranging from 1-100 $\mu \mathrm{M}$ were used. Sodium cacodylate buffer was used at $\mathrm{pH}$ 6.1, which is essential for experiments using RNA. During the DSF experiment, the temperature was increased from 4 to $95^{\circ} \mathrm{C}$ at an increment of $0.2^{\circ} \mathrm{C}$ with an equilibration time of $5 \mathrm{~s}$ at each temperature prior to measurement.

For each FID assays, a $1 \mathrm{ml}$ solution containing the double-stranded CUG RNA ( $0.25 \mathrm{M})$ and the indicator Thiazole Orange $(0.75 \mathrm{M})$ was prepared using cacodylate buffer $(\mathrm{pH} .7 .4)$. The solution was incubated at 
$25 \mathrm{C}$ for 10 min before measuring the initial fluorescence spectrum. Then, aliquots of the tested compound (peptide $0.125 \mathrm{mM}$ solutions in DMSO) were subsequently added up to saturation. After each addition, the cuvette was rigorously homogenized and let to stand for five min prior to measure the emission spectrum. All the experiments were performed at least in duplicate to ensure the reproducibility of the data. The values calculated for Ka and their associated errors come from averaging. The equipment used was a modular PTI fluorescence instrument (slit widths of $5 \mathrm{~nm}$ and power of $750 \mathrm{mV}$ ). The measurements were carried out using $1 \mathrm{ml}$ quartz cuvettes with a path-length of $1 \mathrm{~cm}$. The Thiazole Orange was excited at $495 \mathrm{~nm}$ and the emission spectrum was registered between 505 and $650 \mathrm{~nm}$. The data was analyzed with the software HypSpec ${ }^{50}$. Once having established an initial equilibrium model for the interaction between the CUG, the TO and the peptide, the software applies an iterative algorithm in order to fit the experimental data to the proposed model, enabling the determination of the affinity constants ${ }^{39,51}$.

\section{Molecular modeling methods}

The molecular structures of 79-82 peptides were created and prepared (capped with ACE and NME blocking groups and chirality modified) using the AMBER tleap module ${ }^{52}$. Molecular dockings were conducted using MOE 2019.01 software (Chemical Computing Group, Montreal, QC). The molecular structure of r(CUG) 16 hairpin was previously modeled by homology ${ }^{53}$ using ModeRNA software ${ }^{54}$, and its stabilization was consequently studied through molecular dynamics simulations. Peptide structures were modeled in MOE and confronted with RNA, following the available induced fit protocol to consider both ligand and RNA as flexible structures. Triangle matcher algorithm was defined for placement, and binding energies were quantitatively estimated by GBVI/WSA dG rescoring function. The dynamic behavior of peptide-RNA complexes was assessed using molecular dynamics simulations using AMBER16 (University of California, San Francisco, CA). Molecular systems were prepared and solvated in TIP3P water solvent using tleap module. After solvent relaxation, constraining the RNA-peptide structure with a force constant of $2.0 \mathrm{kcal} \cdot \mathrm{mol}-1 \cdot \AA-2$, the system was slowly heated at $300 \mathrm{~K}$ in $1 \mathrm{~ns}$. A density equilibration stage under NPT ensemble $(P=1 \mathrm{bar})$ preceded the production stage, which was conducted at NVT conditions at $300 \mathrm{~K}$ during $10 \mathrm{~ns}$. All simulations were performed under periodic boundary conditions, using the Particle Mesh Ewald (PME) to describe electrostatic interactions and SHAKE algorithm. The time step was fixed to $2 \mathrm{fs}$. Trajectory analysis, including the calculation of helical parameters, were conducted using cpptraj and considering the last 5 ns of the simulation ${ }^{55}$.

\section{Statistical methods}

We assumed in all our experiments that parameters follow a normal distribution. In the molecular, functional, and histological analyses, pairs of samples were compared using two-tailed t-tests $(a=0.05)$, applying Welch's correction when necessary. The statistical significance of the differences for all data reported can be found in Supplementary Table 2. The sample size is stated in each figure. In the functional analysis of flight ability of the flies, Fisher's exact test was applied to compare percentages of flies able to fly with those unable. 


\section{Declarations}

\section{Acknowledgments}

This work was supported by research grants "Analysis of the structure-activity relationships of antimyotonic dystrophy hexapeptides" from "Telemaratón Todos Somos Raros, Todos Somos Únicos" to B.L., and project PI13/00386 from the Instituto de Salud Carlos III, which included funds from the FEDER program, to M.P.A. We also thank a generous gift from Gestion de Bienes y Servicios that partially supported A.S.R. and the collaborative project 21-Peptide-DM-Garcia-España-Artero-2017-B (VLCBIOCLINIC program) to E.G.E. and R.A. A.B. was supported by a postdoctoral fellowship (APOSTD2017/077).

\section{Author contributions}

A.R. Investigation, formal analysis, visualization, writing-original draft.

A.B. Formal analysis, visualization, writing-review and editing.

B.LL. Conceptualization, formal analysis, project administration, supervision, funding acquisition.

R.E.T. Investigation, formal analysis, visualization.

I.P. Investigation, formal analysis, visualization.

E.G.E. Investigation, formal analysis, visualization.

R.A. Conceptualization, resources, supervision, writing-review and editing.

M.P.A. Conceptualization, funding acquisition, project administration, supervision.

\section{Additional Information}

The authors declare no competing interests.

\section{References}

1. Ashizawa, T. et al. Consensus-based care recommendations for adults with myotonic dystrophy type 1. Neurol. Clin. Pract. 8, 507-520 (2018).

2. Brook, J. D. et al. Molecular basis of myotonic dystrophy: expansion of a trinucleotide (CTG) repeat at the $3^{\prime}$ end of a transcript encoding a protein kinase family member. Cel/ 69, 385 (1992).

3. Pettersson, O. J., Aagaard, L., Jensen, T. G. \& Damgaard, C. K. Molecular mechanisms in DM1 - a focus on foci. Nucleic Acids Res. 43, 2433-2441 (2015).

4. Lee, K.-Y. et al. Compound loss of muscleblind-like function in myotonic dystrophy. EMBO Mol. Med. 5, 1887-1900 (2013). 
5. Kanadia, R. N. et al. Reversal of RNA missplicing and myotonia after muscleblind overexpression in a mouse poly(CUG) model for myotonic dystrophy. Proc. Natl. Acad. Sci. U. S. A. 103, 11748-11753 (2006).

6. Chamberlain, C. M. \& Ranum, L. P. W. Mouse model of muscleblind-like 1 overexpression: skeletal muscle effects and therapeutic promise. Hum. Mol. Genet. 21, 4645-4654 (2012).

7. Konieczny, P., Stepniak-Konieczna, E. \& Sobczak, K. MBNL proteins and their target RNAs, interaction and splicing regulation. Nucleic Acids Res. 42, 10873-10887 (2014).

8. Sznajder, Ł. J. et al. Mechanistic determinants of MBNL activity. Nucleic Acids Res. 44, 1032610342 (2016).

9. Batra, R. et al. Loss of MBNL leads to disruption of developmentally regulated alternative polyadenylation in RNA-mediated disease. Mol. Cell 56, 311-322 (2014).

10. Ozimski, L. L., Sabater-Arcis, M., Bargiela, A. \& Artero, R. The hallmarks of myotonic dystrophy type 1 muscle dysfunction. Biological Reviews (2020) doi:10.1111/brv.12674.

11. Jones, K. et al. GSK3 $\beta$ mediates muscle pathology in myotonic dystrophy. J. Clin. Invest. 122, 44614472 (2012).

12. Sabater-Arcis, M., Bargiela, A., Furling, D. \& Artero, R. miR-7 Restores Phenotypes in Myotonic Dystrophy Muscle Cells by Repressing Hyperactivated Autophagy. Mol. Ther. Nucleic Acids 19, 278292 (2020).

13. Bargiela, A. et al. Increased autophagy and apoptosis contribute to muscle atrophy in a myotonic dystrophy type 1 Drosophila model. Dis. Model. Mech. 8, 679-690 (2015).

14. Konieczny, P. et al. Myotonic dystrophy: candidate small molecule therapeutics. Drug Discov. Today 22, 1740-1748 (2017).

15. Overby, S. J., Cerro-Herreros, E., Llamusi, B. \& Artero, R. RNA-mediated therapies in myotonic dystrophy. Drug Discov. Today 23, 2013-2022 (2018).

16. Chen, G. et al. Phenylbutazone induces expression of MBNL1 and suppresses formation of MBNL1CUG RNA foci in a mouse model of myotonic dystrophy. Sci. Rep. 6, 25317 (2016).

17. Cerro-Herreros, E. et al. Therapeutic Potential of AntagomiR-23b for Treating Myotonic Dystrophy. Molecular Therapy - Nucleic Acids 21, 837-849 (2020).

18. Zhang, F. et al. A flow cytometry-based screen identifies MBNL1 modulators that rescue splicing defects in myotonic dystrophy type I. Hum. Mol. Genet. 26, 3056-3068 (2017).

19. Bargiela, A. et al. Increased Muscleblind levels by chloroquine treatment improve myotonic dystrophy type 1 phenotypes in in vitro and in vivo models. Proc. Natl. Acad. Sci. U. S. A. 116, 25203-25213 (2019).

20. Ramon-Duaso, C. et al. Protective effects of mirtazapine in mice lacking the Mbnl2 gene in forebrain glutamatergic neurons: Relevance for myotonic dystrophy 1. Neuropharmacology 108030 (2020).

21. Chakraborty, M. et al. Daunorubicin reduces MBNL1 sequestration caused by CUG-repeat expansion and rescues cardiac dysfunctions in a Drosophila model of myotonic dystrophy. Dis. Model. Mech. 
11, (2018).

22. García-López, A., Llamusí, B., Orzáez, M., Pérez-Payá, E. \& Artero, R. D. In vivo discovery of a peptide that prevents CUG-RNA hairpin formation and reverses RNA toxicity in myotonic dystrophy models. Proc. Natl. Acad. Sci. U. S. A. 108, 11866-11871 (2011).

23. Arandel, L. et al. Immortalized human myotonic dystrophy muscle cell lines to assess therapeutic compounds. Dis. Model. Mech. 10, 487-497 (2017).

24. Du, H. et al. Aberrant alternative splicing and extracellular matrix gene expression in mouse models of myotonic dystrophy. Nat. Struct. Mol. Biol. 17, 187-193 (2010).

25. Osborne, R. J. et al. Transcriptional and post-transcriptional impact of toxic RNA in myotonic dystrophy. Hum. Mol. Genet. 18, 1471-1481 (2009).

26. Nakamori, M. et al. Splicing biomarkers of disease severity in myotonic dystrophy. Ann. Neurol. 74, 862-872 (2013).

27. Charizanis, K. et al. Muscleblind-like 2-mediated alternative splicing in the developing brain and dysregulation in myotonic dystrophy. Neuron 75, 437-450 (2012).

28. Kalsotra, A. et al. A postnatal switch of CELF and MBNL proteins reprograms alternative splicing in the developing heart. Proc. Natl. Acad. Sci. U. S. A. 105, 20333-20338 (2008).

29. Warf, M. B., Nakamori, M., Matthys, C. M., Thornton, C. A. \& Berglund, J. A. Pentamidine reverses the splicing defects associated with myotonic dystrophy. Proc. Natl. Acad. Sci. U. S. A. 106, 1855118556 (2009).

30. Cerro-Herreros, E. et al. miR-23b and miR-218 silencing increase Muscleblind-like expression and alleviate myotonic dystrophy phenotypes in mammalian models. Nat. Commun. 9, 2482 (2018).

31. deLorimier, E. et al. Modifications to toxic CUG RNAs induce structural stability, rescue mis-splicing in a myotonic dystrophy cell model and reduce toxicity in a myotonic dystrophy zebrafish model. Nucleic Acids Res. 42, 12768-12778 (2014).

32. Squillace, R. M., Chenault, D. M. \& Wang, E. H. Inhibition of muscle differentiation by the novel muscleblind-related protein CHCR. Dev. Biol. 250, 218-230 (2002).

33. Timchenko, N. A., lakova, P., Cai, Z. J., Smith, J. R. \& Timchenko, L. T. Molecular basis for impaired muscle differentiation in myotonic dystrophy. Mol. Cell. Biol. 21, 6927-6938 (2001).

34. Loro, E. et al. Normal myogenesis and increased apoptosis in myotonic dystrophy type-1 muscle cells. Cell Death Differ. 17, 1315-1324 (2010).

35. Klionsky, D. J. et al. Guidelines for the use and interpretation of assays for monitoring autophagy. Autophagy 8, 445-544 (2012).

36. Llamusi, B. et al. Muscleblind, BSF and TBPH are mislocalized in the muscle sarcomere of a Drosophila myotonic dystrophy model. Dis. Model. Mech. 6, 184-196 (2013).

37. Garcia-Lopez, A. et al. Genetic and chemical modifiers of a CUG toxicity model in Drosophila. PLoS One 3, e1595 (2008). 
38. Silvers, R., Keller, H., Schwalbe, H. \& Hengesbach, M. Differential scanning fluorimetry for monitoring RNA stability. Chembiochem 16, 1109-1114 (2015).

39. Asare-Okai, P. N. \& Chow, C. S. A modified fluorescent intercalator displacement assay for RNA ligand discovery. Anal. Biochem. 408, 269-276 (2011).

40. Tran, P. L. T., Largy, E., Hamon, F., Teulade-Fichou, M.-P. \& Mergny, J.-L. Fluorescence intercalator displacement assay for screening G4 ligands towards a variety of G-quadruplex structures. Biochimie 93, 1288-1296 (2011).

41. Jamieson, A. G., Boutard, N., Sabatino, D. \& Lubell, W. D. Peptide Scanning for Studying StructureActivity Relationships in Drug Discovery. Chemical Biology \& Drug Design vol. 81 148-165 (2013).

42. Lau, J. L. \& Dunn, M. K. Therapeutic peptides: Historical perspectives, current development trends, and future directions. Bioorg. Med. Chem. 26, 2700-2707 (2018).

43. Fosgerau, K. \& Hoffmann, T. Peptide therapeutics: current status and future directions. Drug Discov. Today 20, 122-128 (2015).

44. Parthasarathy, A., Anandamma, S. K. \& Kalesh, K. A. The Medicinal Chemistry of Therapeutic Peptides: Recent Developments in Synthesis and Design Optimizations. Curr. Med. Chem. 26, 23302355 (2019).

45. Guidotti, G., Brambilla, L. \& Rossi, D. Cell-Penetrating Peptides: From Basic Research to Clinics. Trends Pharmacol. Sci. 38, 406-424 (2017).

46. Boisguérin, P. et al. Delivery of therapeutic oligonucleotides with cell penetrating peptides. Adv. Drug Deliv. Rev. 87, 52-67 (2015).

47. Reese, H. R., Shanahan, C. C., Proulx, C. \& Menegatti, S. Peptide science: A 'rule model' for new generations of peptidomimetics. Acta Biomater. 102, 35-74 (2020).

48. Selma-Soriano, E., Artero, R. \& Llamusi, B. Optical Cross-Sectional Muscle Area Determination of Drosophila Melanogaster Adult Indirect Flight Muscles. J. Vis. Exp. (2018) doi:10.3791/56179.

49. González, À. L. et al. In silico discovery of substituted pyrido[2,3-d]pyrimidines and pentamidine-like compounds with biological activity in myotonic dystrophy models. PLoS One 12, e0178931 (2017).

50. Gans, P., Sabatini, A. \& Vacca, A. Investigation of equilibria in solution. Determination of equilibrium constants with the HYPERQUAD suite of programs. Talanta 43, 1739-1753 (1996).

51. Boger, D. L. \& Tse, W. C. Thiazole orange as the fluorescent intercalator in a high resolution fid assay for determining DNA binding affinity and sequence selectivity of small molecules. Bioorg. Med. Chem. 9, 2511-2518 (2001).

52. Case, D. A. et al. The Amber biomolecular simulation programs. J. Comput. Chem. 26, 1668-1688 (2005).

53. González, À. L., Teixidó, J., Borrell, J. I. \& Estrada-Tejedor, R. On the Applicability of Elastic Network Models for the Study of RNA CUG Trinucleotide Repeat Overexpansion. PLoS One 11, e0152049 (2016). 
54. Rother, M., Rother, K., Puton, T. \& Bujnicki, J. M. ModeRNA: a tool for comparative modeling of RNA 3D structure. Nucleic Acids Res. 39, 4007-4022 (2011).

55. Roe, D. R. \& Cheatham, T. E., 3rd. PTRAJ and CPPTRAJ: Software for Processing and Analysis of Molecular Dynamics Trajectory Data. J. Chem. Theory Comput. 9, 3084-3095 (2013).

\section{Figures}
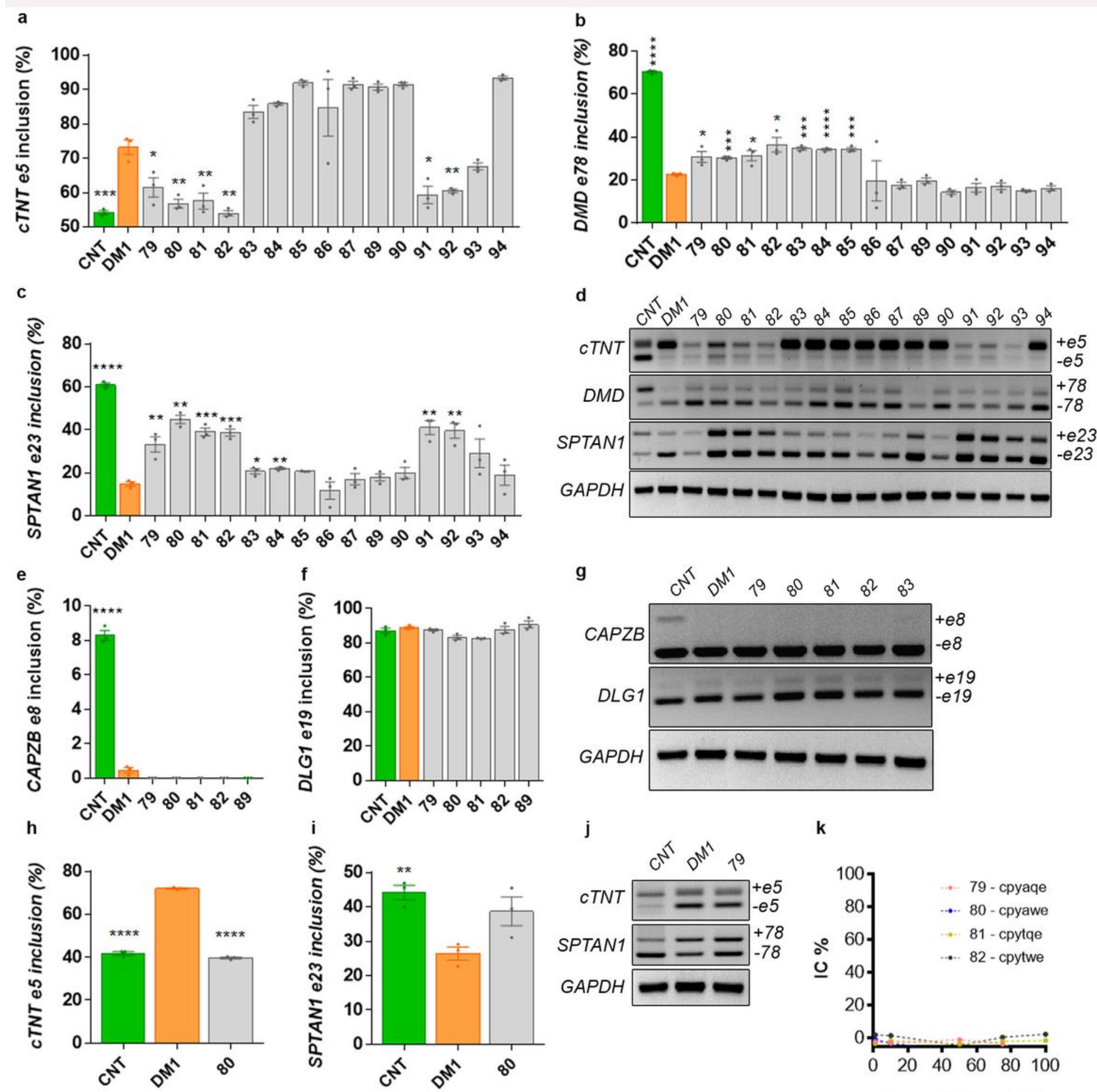

g
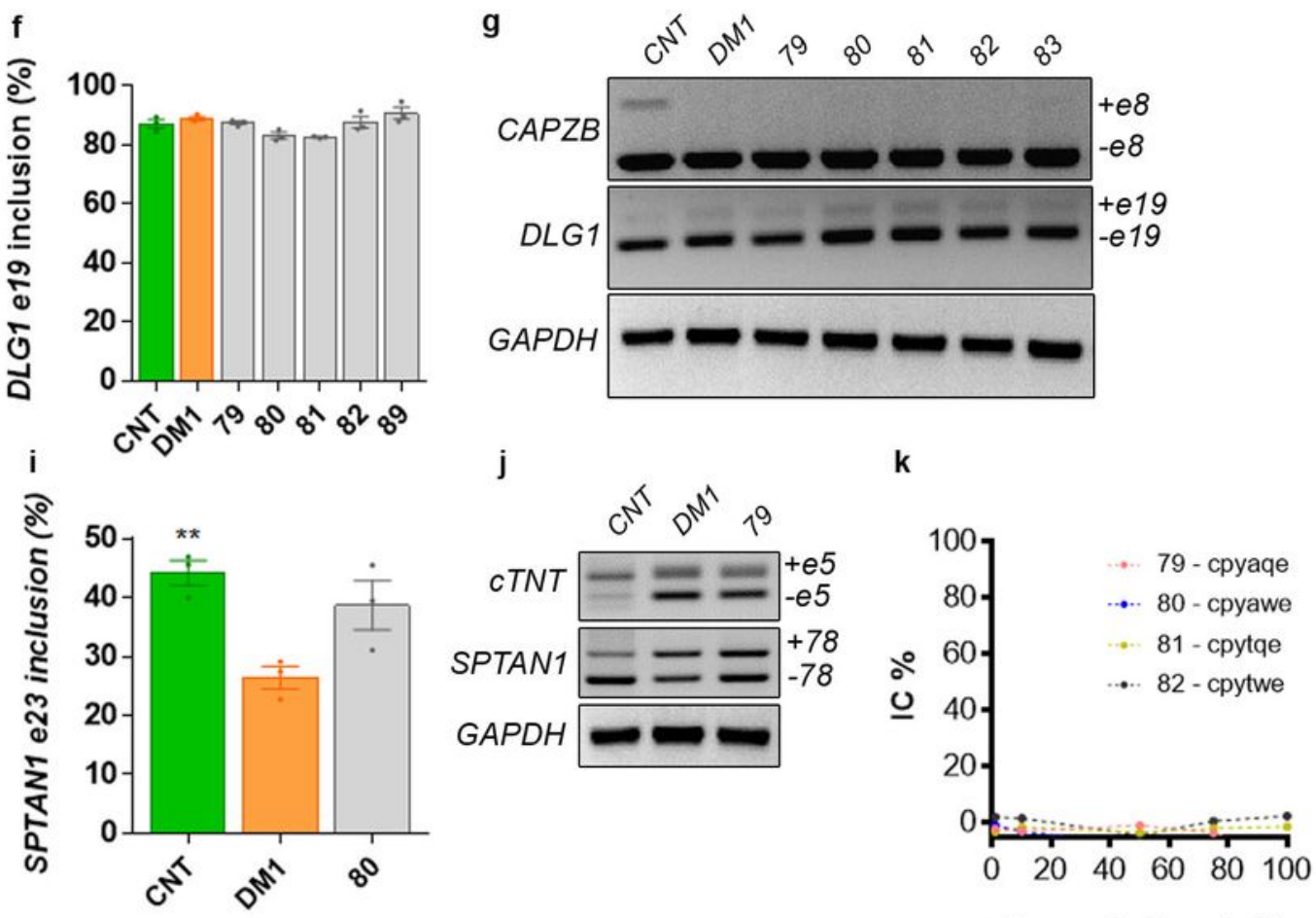

Concentration $(\mu \mathrm{M})$ 


\section{Figure 1}

Peptides 79, 80, 81, and 82 rescued MBNL-dependent mis-splicing events in DM1 myoblasts. Control (CNT, with no DMSO) and DM1 myoblasts (96 h of differentiation) were treated with $10 \mu \mathrm{M}$ of the indicated peptides or DMSO (0.1\%) for $48 \mathrm{~h}$ and the percentage of inclusion of cTNT exon 5 (a), DMD exon 78 (b), and SPTAN1 exon 23 (c) was determined. (d) Representative $2 \%$ agarose gels showing semiquantitative RT-PCR amplicons with or without the indicated exons and GAPDH internal control. None of the tested peptides induced changes in the splicing of the CAPZB gene, altered in DM1 and regulated by the CELF1 protein $(e, g)$ or in the splicing of the DLG1 gene, which remains unchanged in DM1 $(f, g)$. The inclusion of exon 5 of the cTNT gene, regulated by MBNL1, $(h, j)$ and the inclusion of the exon 23 of the gene SPTAN1, regulated by MBNL2 (i,j), did not respond to peptide 80 in CNT myoblasts, thus supporting the specificity of its activity. None of the selected compounds was toxic in control myoblasts, even at a concentration tenfold higher than the active dose (k). ${ }^{*} p<0.05,{ }^{* \star} p<0.01,{ }^{\star \star *} p<0.001$, $\star \star \star \star * p<0.0001$, according to Student's t-test. 
a

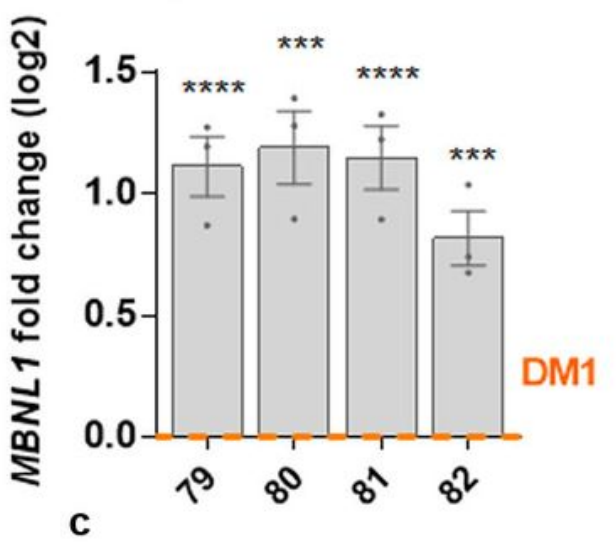

b

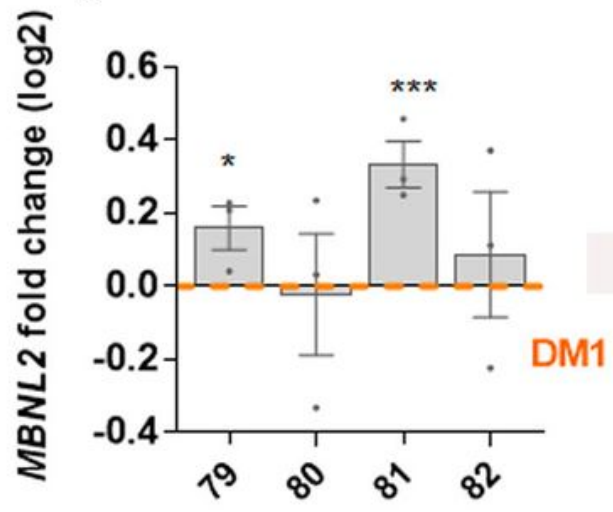

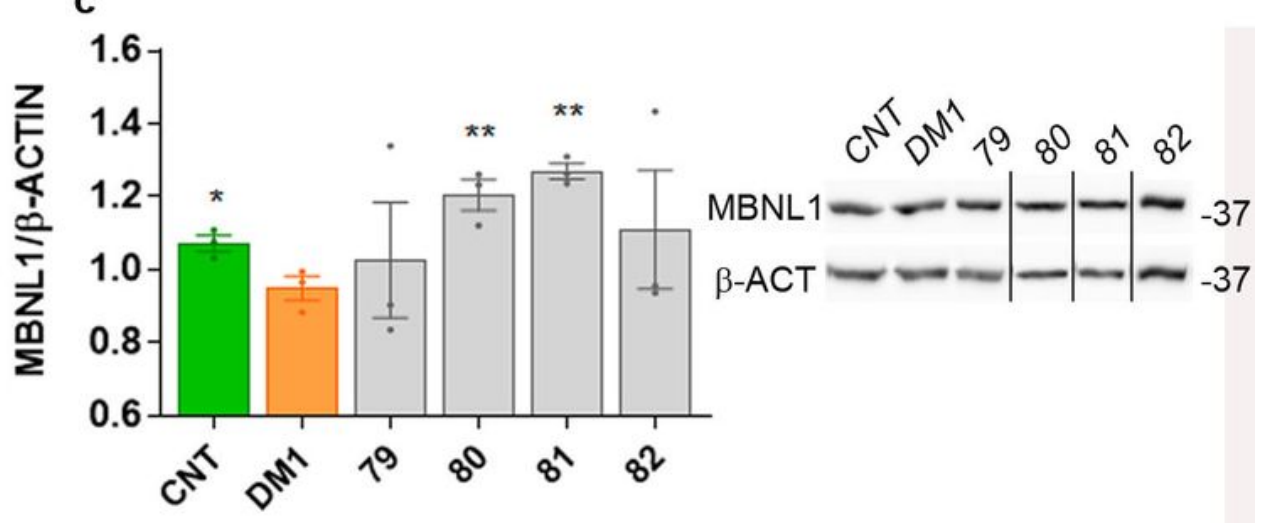

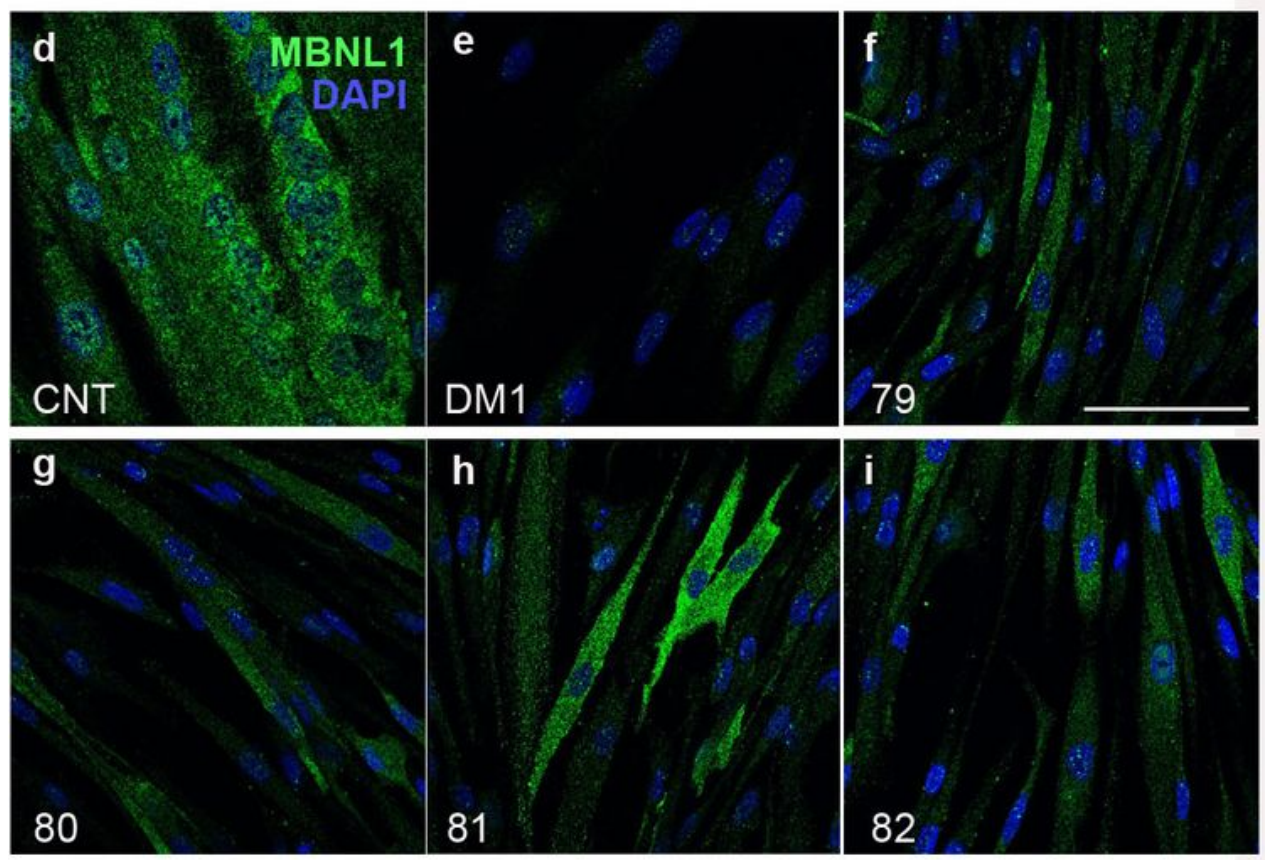

Figure 2

Candidate peptides enhanced the expression of MBNL proteins in a DM1 cell model. Quantification by RTqPCR of relative expression of MBNL1 and MBNL2 transcripts in DM1 cells differentiated for four days and treated with the four candidate peptides. Levels were referenced to GAPDH as endogenous control $(a, b)$. Western blot quantification relative to beta-Actin confirmed a significant increase in the levels of MBNL1 protein in the cells treated with the 80 and 81 peptides. Black lines indicate those lanes cropped 
from different blots (c). The molecular weight marker band at $37 \mathrm{kDa}$ is indicated to the right of the blot. Confocal microscopy micrographs of control (CNT, d; without DMSO) and DM1 myoblasts (e-i) treated with vehicle (DMSO $0.1 \%$; e) or the indicated peptides (f-i) stained for MBNL1 (green channel) and DAPI. All images were taken at the same settings. Note the general increase in the intensity of MBNL1 fluorescence in (f-i) panels. ${ }^{\star} p<0.05,{ }^{* \star} p<0.01,{ }^{\star \star \star} p<0.001$ according to Student's t-test.Scale bar corresponds to 100 microns.

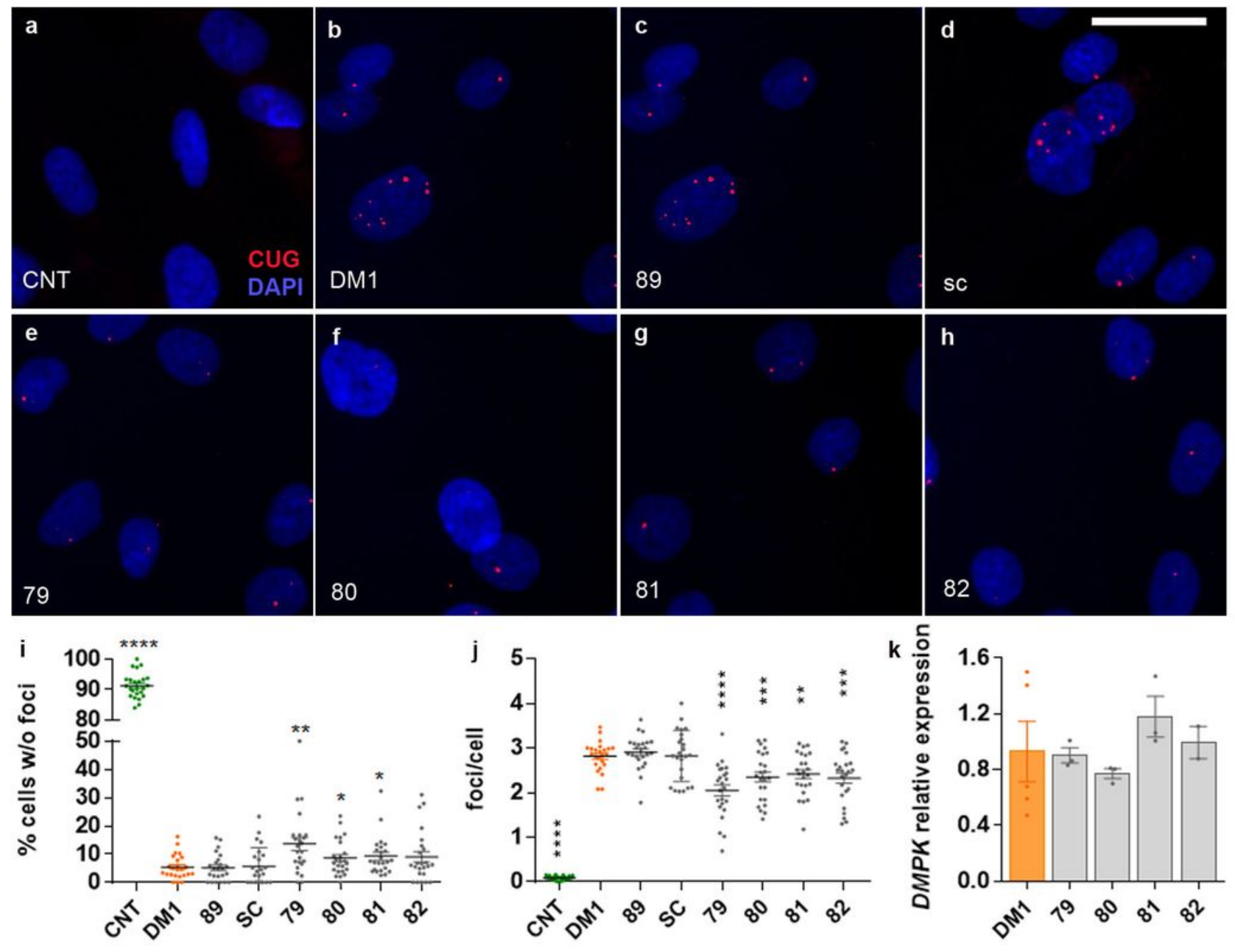

Figure 3

Candidate peptides reduced ribonuclear foci in DM1 cells. Representative micrographs of foci in control (CNT, a; without DMSO), DM1 (DMSO 0.1\%, b), and DM1 cells treated with negative control (c,d) or candidate peptides (e-h) obtained with an IN Cell Analyzer high-content imaging system. Accumulation of mutant transcripts was detected using fluorescent in situ hybridization (FISH) with a Cy3-labeled RNA probe (red dots). Nuclei were counterstained with Hoechst (blue). Quantification of the images revealed that peptides 79,80 , and 81 significantly increased the percentage of cells without foci (i) and reduced the number of foci per cell, in this case including peptide $82(j)$. The observed reduction in mutant DMPK accumulation was not due to the repression of the DMPK gene expression itself, which was quantified by real-time PCR using primers against a non-repetitive sequence and was found not significantly different 
from DM1 controls $(k) .{ }^{*} p<0.05,{ }^{* \star} p<0.01,{ }^{* \star} p<0.001$ according to Student's t-test. Scale bar (a-h) measures 20 microns.
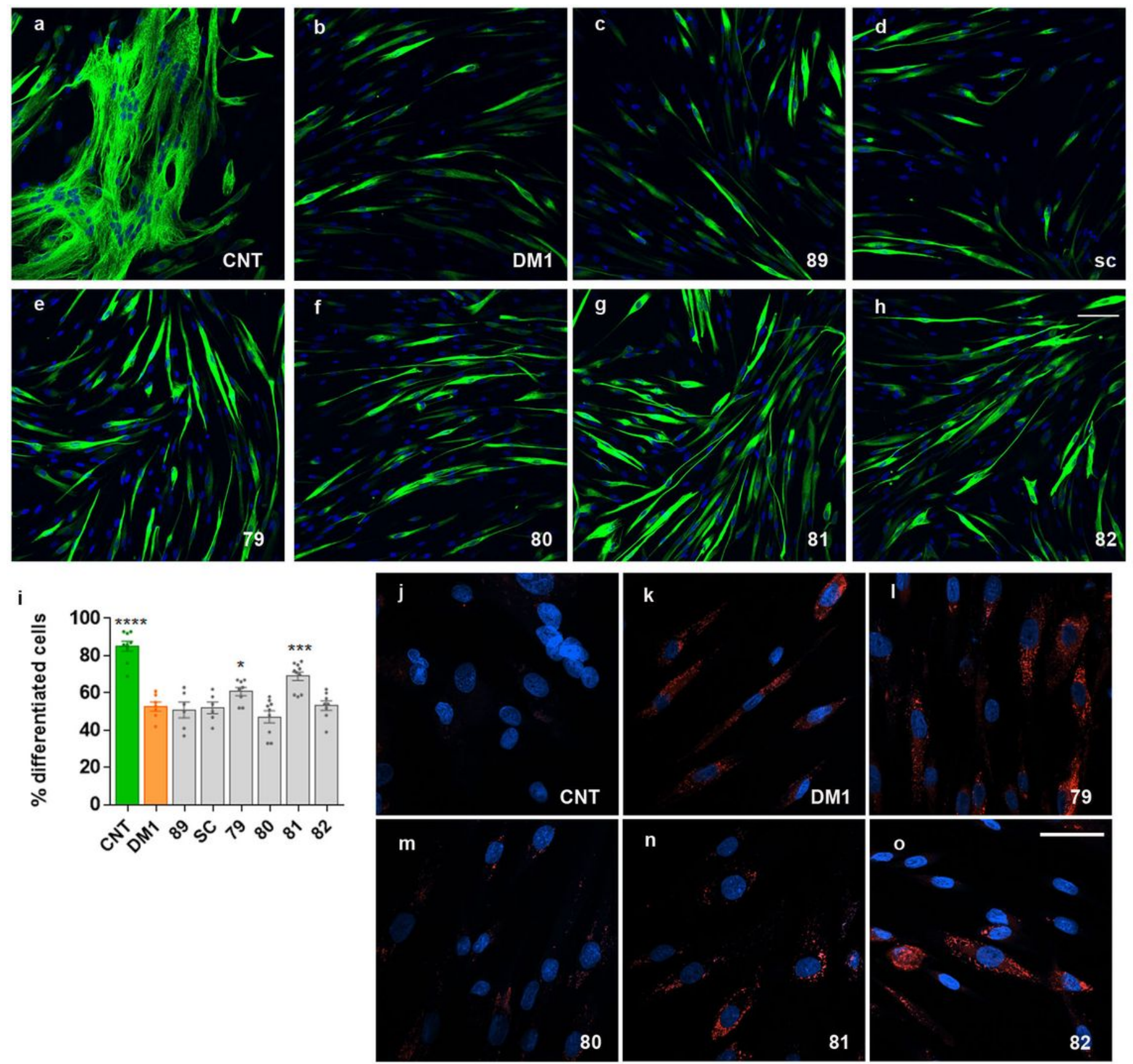

\section{Figure 4}

Differentiation delay and autophagy hyperactivation were rescued by peptides. Representative micrographs of control (CNT, a; without DMSO), DM1 (DMSO 0.1\%; b) and DM1 cells treated with negative control $(c, d)$ or candidate peptides $(e-h)$ stained for Desmin (green signal) as a marker of myogenic differentiation. Cells differentiated in DMEM for seven days and were treated with 10 microM of the indicated peptides. Quantification of the number of Desmin-positive cells relative to the total number of cells (i) revealed a significant increase in myogenic cells when treated with peptides 79 and 81. Human myoblasts stained with LysoTracker (red fluorescence; j-o). Autolysosomal labeling is 
observed in DM1 myoblasts (DM1) but not in controls (CNT), denoting increased autophagy in DM1 cells. Cells treated with peptide 80 showed a general reduction of the signal from auto lysosomal vesicles while for cells treated with peptides 81 and 82, an increase in the number of cells without autophagic vesicles around the nucleus was observed. (a-h, j-o) Nuclei were counterstained with Hoechst 33258 (blue). ${ }^{*} \mathrm{p}<0.05,{ }^{* *} \mathrm{p}<0.01,{ }^{* \star *} \mathrm{p}<0.001$ according to Student's t-test. Scale bar corresponds to 100 (a-h) and 40 microns (j-o).
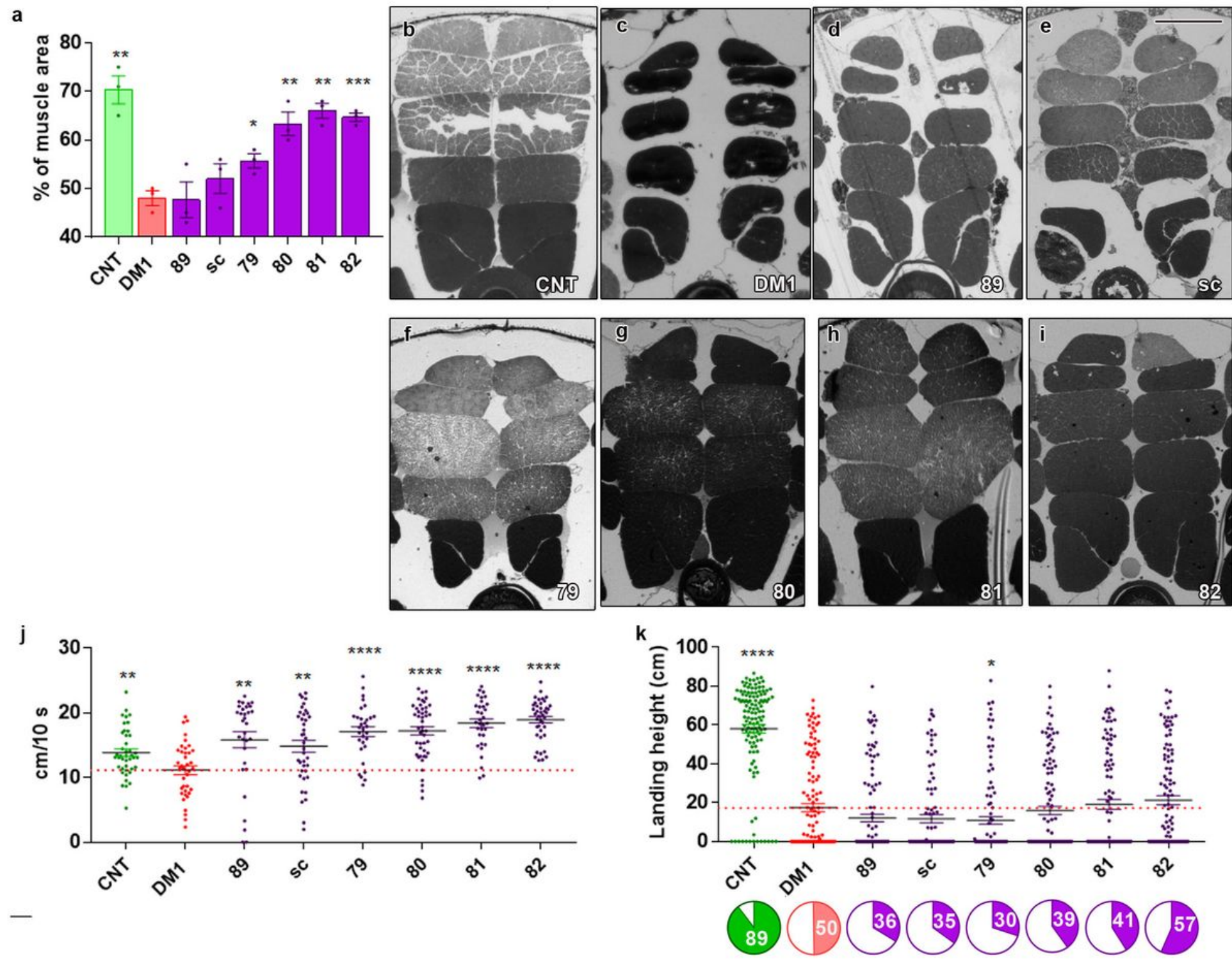

\section{Figure 5}

Candidate peptides rescued muscle atrophy in a Drosophila DM1 model. Quantification of indirect flight muscle (IFM) cross-sectional area (a) of control (CNT), DM1 flies taking vehicle (DMSO) and DM1 flies taking negative controls ( 89 or $\mathrm{sc}$ ) or the indicated candidate peptides. All four candidate peptides increased the mean muscle area relative to DMSO-treated flies $(n=6)$. $(b-i)$ Representative bright-field microscope images of transversal sections of resin-embedded adult IFM of Mhc-Gal4 UAS-i(CTG)480 (Rec2) heterozygous flies treated with DMSO $(0.01 \%)$ or with peptides $79-82(10 \mu \mathrm{M})$ that were used to 
generate the data shown in (a). Muscle recovery at the histological level leads to functional improvements. The climbing assay $(n=30 ; j)$ showed a significant increase in the speed of the treated flies compared to the untreated ones, calculated as the distance traveled in $10 \mathrm{~s}$. In the flight assay $(n=100 ; k)$, the average value of the landing height did not significantly improve, but there was a clear increase in the percentage of flies that were able to fly (colored sectors in pie charts underneath the graph), especially for flies treated with peptide $82 .{ }^{*} p<0.05,{ }^{*} p<0.01,{ }^{* \star} p<0.001$ according to Student's $t$-test. Scale bar for b-i panels measures 100 microns.
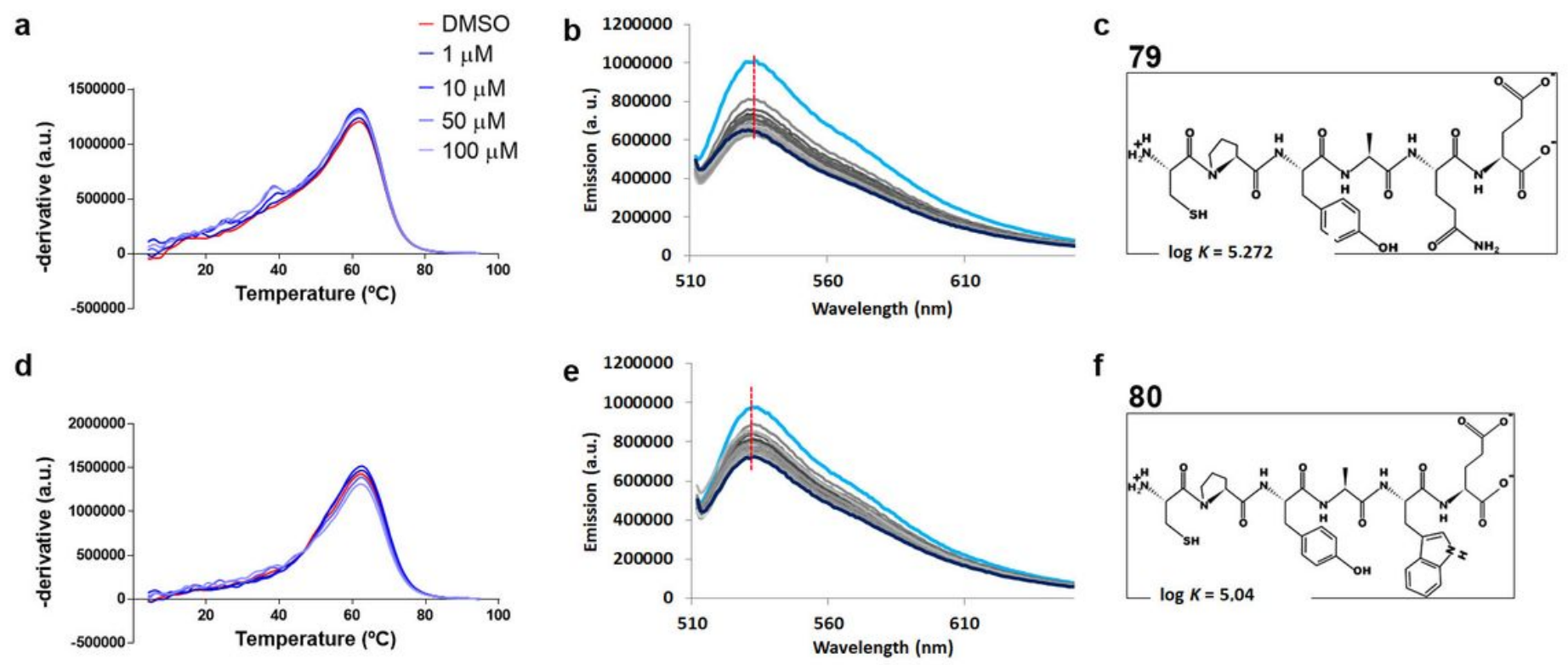

f
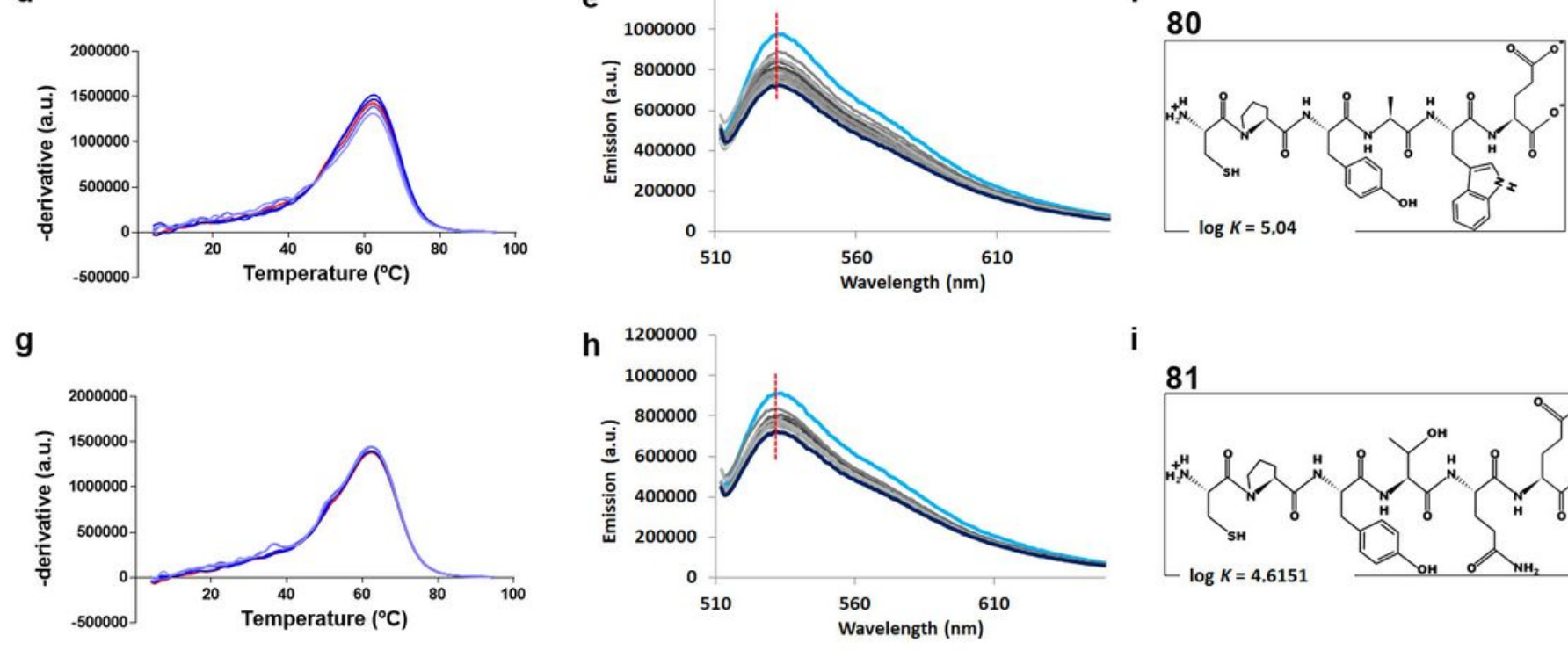

i

81
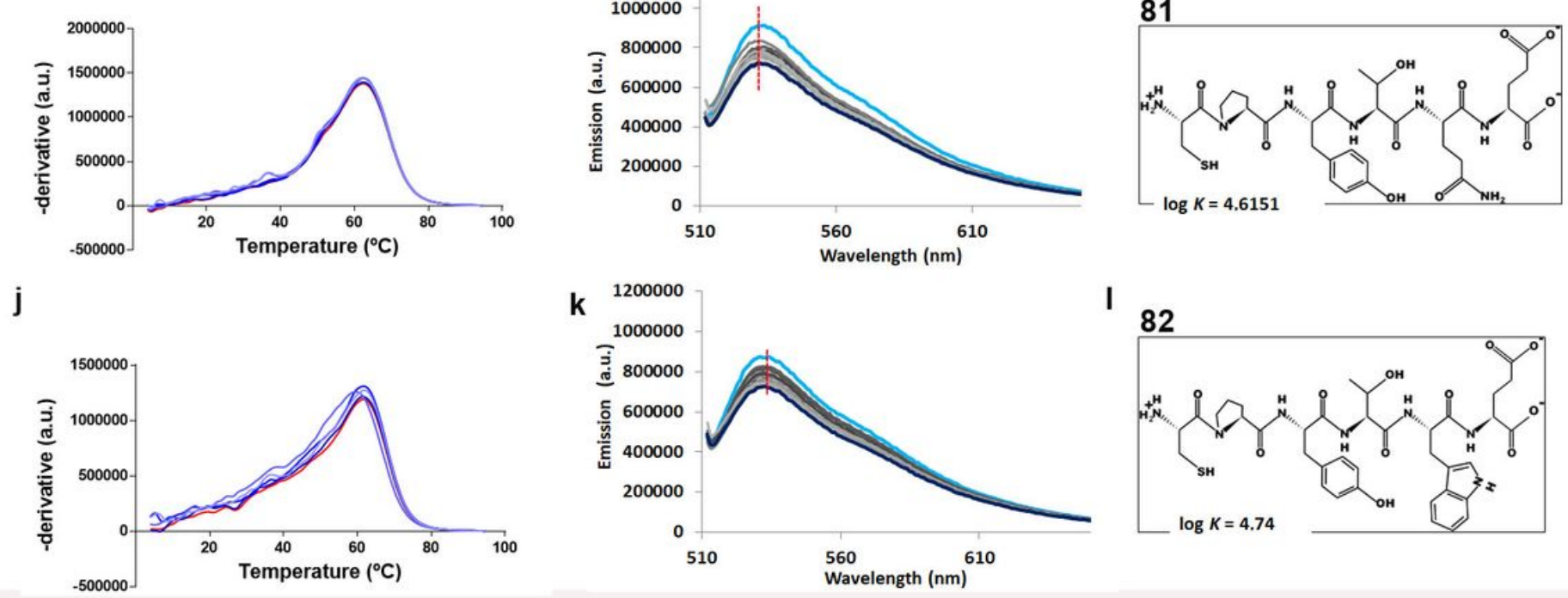

82

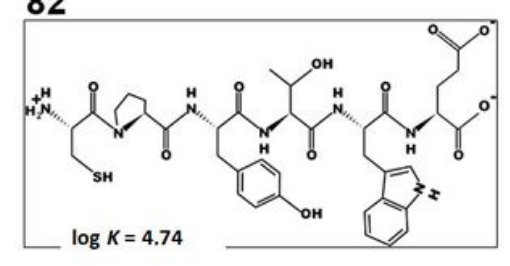

Figure 6

Candidate peptides interact with CUG RNA without changing its secondary structure. The graphs show the first derivatives of RiboGreen fluorescence (DSF assay) versus temperature $(a, d, g, j)$ and Thiazole orange fluorescence titration experiments (b,e,h,k; FID assay) for peptides 79 (a,b,c), 80 (d,e,f), 81 (g,h,i) and $82(j, k, l)$. DMSO $0.1 \%$ was used as a negative control. In Thiazole orange titrations, the intensity of 
the fluorescence progressively decreased with increasingly higher peptide concentrations (concentrations tested up to $8.5 \mathrm{mM}$ ). The affinity constants $(\mathrm{K})$ were calculated from FID assay data, which indicate that peptide 79 interacts with the (CUG)23 RNA probe with the highest affinity, although differences in binding affinity were low among all four peptides. DSF data also supports the binding of peptides to the RNA probe but did not detect significant changes in its secondary structure.

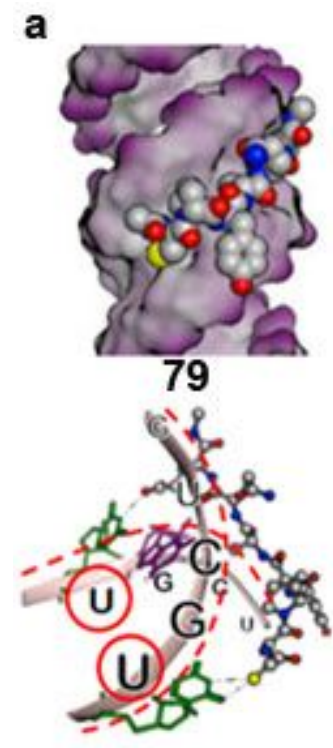

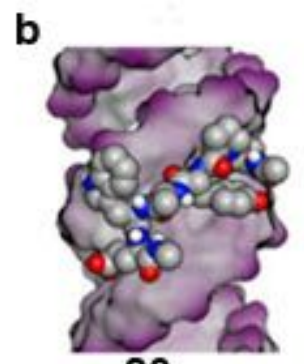

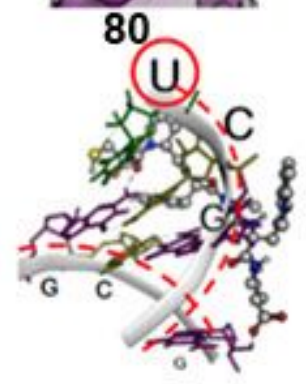

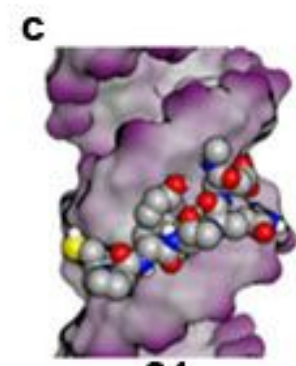

81

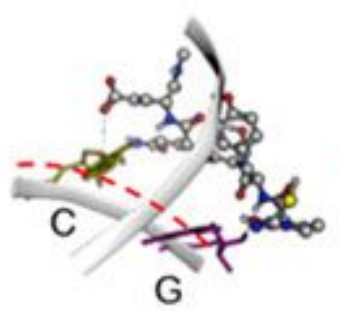

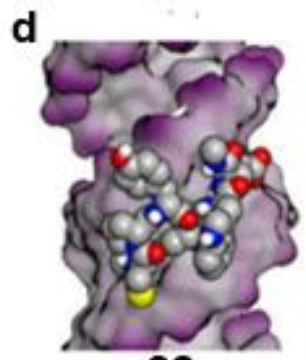

82

\section{Figure 7}

Modeling of the proposed interaction between the candidate peptides and the CUG RNA. Representation of the results obtained by means of a flexible docking directed to the area of the RNA exposed to the solvent. Two different binding mechanisms are proposed: peptides 79 (a) and 80 (b) might interact with the two RNA strands (red lines), recognizing the indicated uracils (red circles); peptides 81 (c) and 82 (d) might interact with only one strand (red line). 


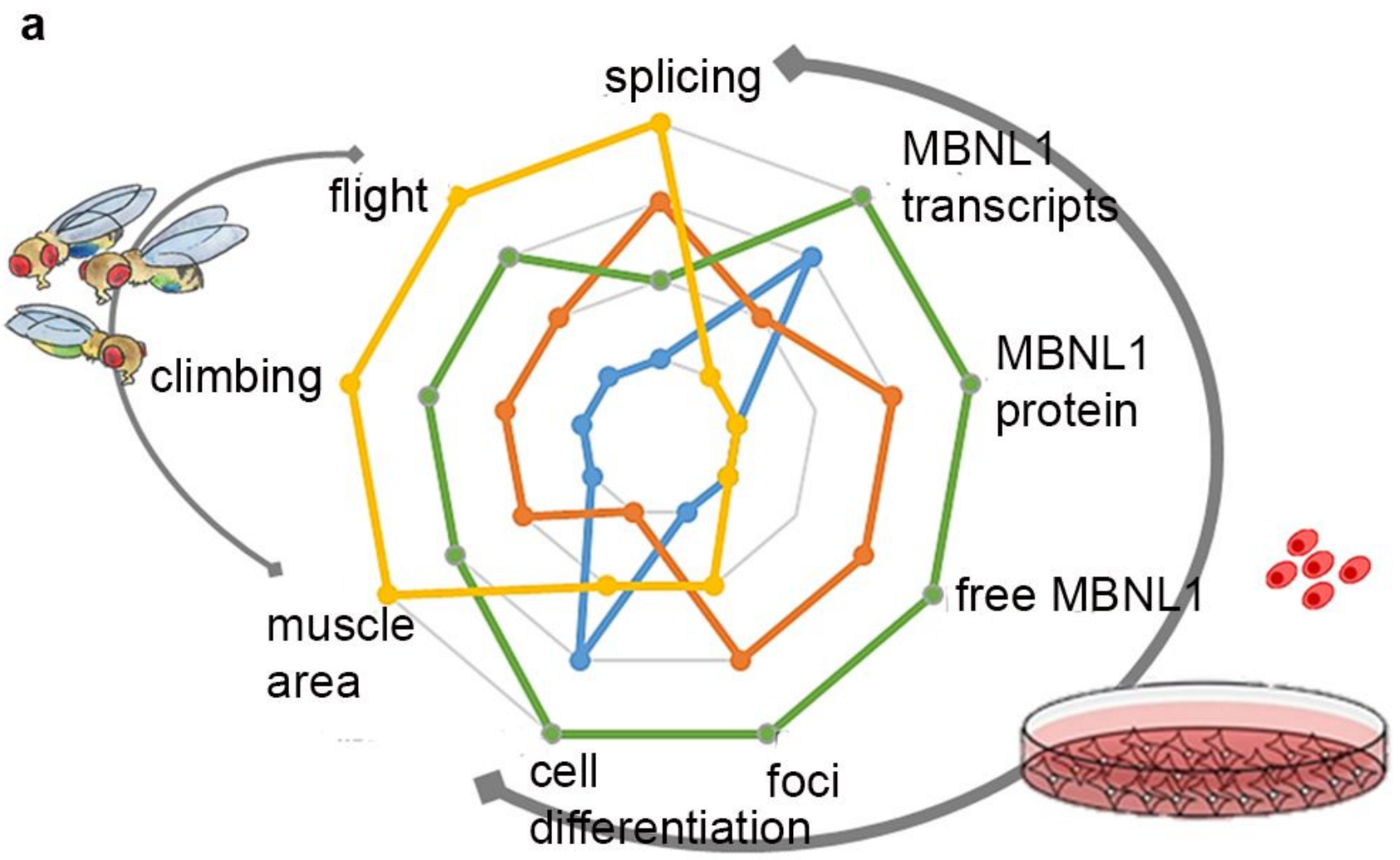

b

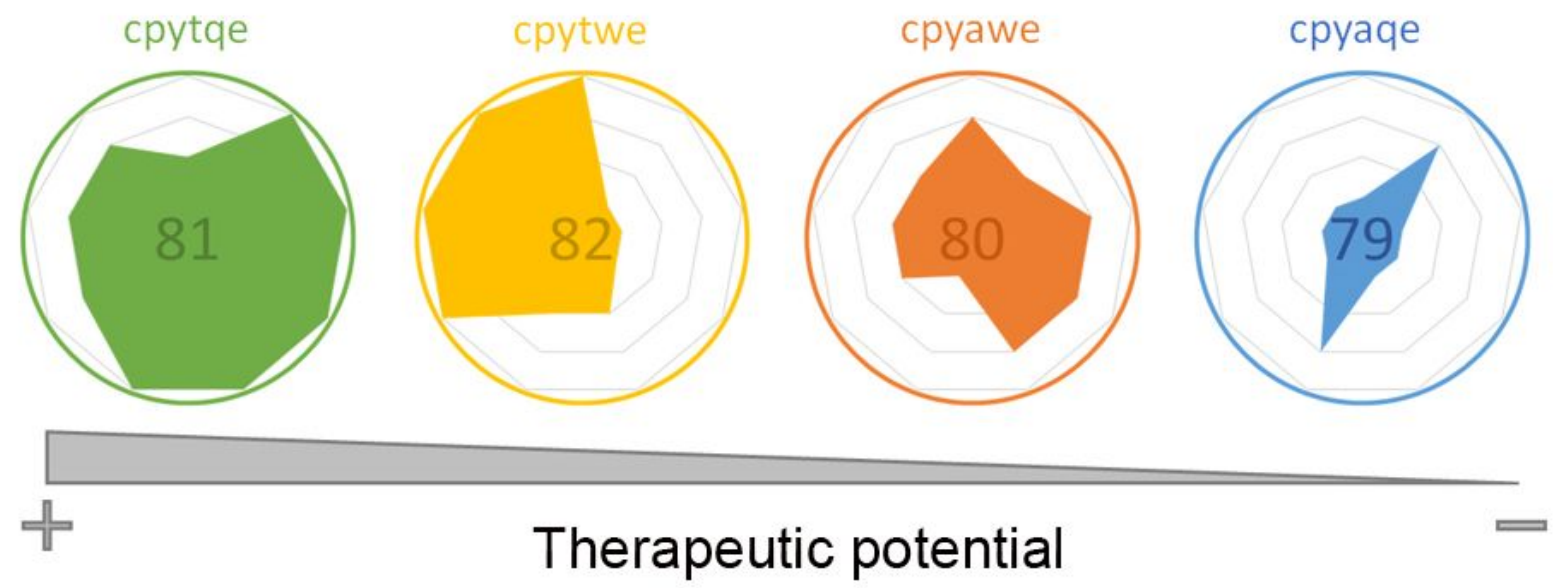

Figure 8

Overall assessment of candidate peptides. Rescues obtained with each peptide (according to the color code shown in b) were converted into a semiquantitative score that reflected the quality of rescue achieved in each of the experimental systems represented (Drosophila and cell model). Scores were inserted into a spider chart, where the internal most polygon indicates no rescue and the outermost 
indicates full rescue (a). The four peptides were sorted according to their therapeutic potential in decreasing order from left to right (b).

\section{Supplementary Files}

This is a list of supplementary files associated with this preprint. Click to download.

- Supplementarylnfo161220.docx 